\title{
PROFILE OF OPERATED ACUTE INTESTINAL OBSTRUCTION PATIENTS AT A TERTIARY HEALTH CARE INSTITUTION
}

\author{
Anoob Mohamed ${ }^{1}$, Niranjan Sahoo², Sushanta Kumar Das ${ }^{3}$, Bhupati Bhusan Das 4 , Sanjeeb Kumar Pradhan ${ }^{5}$, Paresh Kumar Gouda 6 \\ ${ }^{1}$ Resident, Department of General Surgery, MKCG Medical College \& Hospital, Berhampur, Odisha. \\ ${ }^{2}$ Assistant Professor, Department of General Surgery, MKCG Medical College \& Hospital, Berhampur, Odisha. \\ ${ }^{3}$ Professor, Department of General Surgery, MKCG Medical College \& Hospital, Berhampur, Odisha. \\ ${ }_{4}^{4}$ Assistant Professor, Department of General Surgery, MKCG Medical College \& Hospital, Berhampur, Odisha. \\ ${ }^{5}$ Senior Resident, Department of General Surgery, MKCG Medical College \& Hospital, Berhampur, Odisha. \\ ${ }^{6}$ Resident, Department of General Surgery, MKCG Medical College \& Hospital, Berhampur, Odisha.
}

\begin{abstract}
\section{BACKGROUND}

Acute intestinal obstruction is one of the most common surgical emergencies in all age groups, all throughout the world. Whether caused by hernia, postoperative adhesions, neoplasm or related biochemical disturbances, intestinal obstruction of either small bowel or large bowel continues to be a major cause of morbidity and mortality. While the main causes of intestinal obstruction may vary from country to country or among regions within a specific country, knowledge of the local disease patterns and outcomes may raise the index of suspicion, and reduce delays in diagnosis and intervention. This is helpful in settings with limited diagnostic modalities where making a definitive diagnosis may be challenging.

The aim of the study is to analyse the profile of acute intestinal obstruction in operated cases and to analyse the morbidity and mortality factors in these cases.
\end{abstract}

\section{MATERIALS AND METHODS}

A prospective study of 210 patients with features of acute intestinal obstruction and was operated in MKCG Medical College Hospital, Berhampur, Odisha from August 2014 to July 2016.

Inclusion Criteria- Patients in all age groups with clinical picture suggestive of acute intestinal obstruction and underwent laparotomy.

Exclusion Criteria- Patients with provisional diagnosis of intestinal obstruction managed non-surgically.

\section{RESULTS}

The maximum incidence was seen in 41-60 years age group with male predominance. Pain abdomen, constipation, abdominal distension and vomiting were present in majority of cases. Common aetiologies were external hernias, bands, adhesions, volvulus and colon cancer. Small bowel was involved in $64.7 \%$ cases and large bowel in 33.8\%. Resection-anastomosis, adhesiolysis and hernioplasty were the common procedures done. Postoperative complications were seen in $20.47 \%$ of cases. The mortality rate was $8.09 \%$.

\section{CONCLUSION}

Acute intestinal obstruction still remains a major cause of morbidity and mortality. The pattern of obstruction is changing with increasing adhesions and decreasing hernias. The diagnosis of strangulation is still a challenge. Prompt intervention in cases of strangulation is the key for a better outcome. Even though there are great advances in technology at the investigation level, clinical judgement based on history, physical examination and radiography of the abdomen forms the main part in diagnosing a case of acute intestinal obstruction.

\section{KEYWORDS}

Acute Intestinal Obstruction, Hernias, Peritoneal Bands and Adhesions, Volvulus.

HOW TO CITE THIS ARTICLE: Mohamed A, Sahoo N, Das SK, et al. Profile of operated acute intestinal obstruction patients at a tertiary health care institution. J. Evolution Med. Dent. Sci. 2017;6(15):1215-1219, DOI: 10.14260/Jemds/2017/264

\section{BACKGROUND}

Acute intestinal obstruction is one of the most common surgical emergencies in all age groups, all throughout the world. ${ }^{1}$ Mode of presentation is similar in all but cause varies in each age group. The diagnosis and management of the

Financial or Other, Competing Interest: None.

Submission 13-01-2017, Peer Review 06-02-2017,

Acceptance 12-02-2017, Published 20-02-2017.

Corresponding Author:

Dr. Anoob Mohamed,

Resident, Department of General Surgery,

MKCG Medical College \& Hospital,

Berhampur, Odisha-760004.

E-mail: anusm786@yahoo.co.in

DOI: $10.14260 /$ jemds $/ 2017 / 264$

(c) $(1)$ patient with intestinal obstruction is one of the most challenging emergencies that a general surgeon can come across. Whether caused by hernia, postoperative adhesions, neoplasm or related biochemical disturbances, intestinal obstruction of either the small bowel or large bowel continues to be a major cause of morbidity and mortality. ${ }^{2}$ Earlier, mortality and morbidity were very high. Now with better understanding of pathophysiology, improvement in radiological techniques of diagnosis, high degree of refinement in correction of fluid and electrolyte imbalance, introduction of antibiotics for effective bacteriological control, introduction of techniques in gastrointestinal decompression, new surgical principles, as in large bowel obstruction, introduction of on-table lavage, and resection and primary anastomosis, has replaced staged procedures 
and number of days in hospital stay. ${ }^{3}$ Improvement in the field of anaesthesia has also contributed to lowering the morbidity and mortality. This is further influenced by the clinical setting and related comorbidities. ${ }^{4}$ Early diagnosis of obstruction, skilful preoperative and operative management, proper technique during surgery and intensive postoperative treatment yields gratifying results. While the main causes of intestinal obstruction may vary from country to country or among regions within a specific country, knowledge of the local disease patterns and outcomes may raise the index of suspicion, and reduce delays in diagnosis, referral and/or operative intervention. This is especially helpful in settings with limited diagnostic modalities where making a definitive diagnosis may be challenging.

\section{Objective}

To analyse the profile of acute intestinal obstruction in operated cases and to analyse the morbidity and mortality factors in these cases.

\section{MATERIALS AND METHODS}

A prospective study of 210 patients, who presented with features of acute intestinal obstruction and were operated in various surgical units of M.K.C.G Medical College \& Hospital, Berhampur, was conducted during the time period of two years from August 2014 to July 2016. The diagnosis was established on symptomatology, clinical manifestations supported by radiological evidences. The final diagnosis was made in laparotomy and in some after histopathological analysis, wherever required.

Ethical approval to conduct the study was granted by the Institutional Ethics Committee of M.K.C.G Medical College \& Hospital.

\section{Inclusion Criteria}

Patients in all age groups who attended OPD and Emergency Department in MKCG Medical College, Berhampur with history and clinical picture suggestive of acute intestinal obstruction and who underwent laparotomy.

\section{Exclusion Criteria}

Patients with provisional diagnosis of intestinal obstruction and managed non-surgically.

From the time of presentation, the cases were followed up till the time of discharge or death of the patient. Upon admission, thorough history taking and clinical examination was done according to a prepared case proforma in all the patients. All routine blood investigations were done. Anaemia, electrolyte imbalances and renal function assessed and correction done if required. X-ray abdomen erect was done in all cases of acute obstruction except for hernias. USG of abdomen and pelvis done in selected cases. Cases were adequately prepared for emergency laparotomy or hernia repair. Patients with clear-cut signs and symptoms of acute obstruction and also those patients not improving on conservative management were planned for operation after resuscitation. Patients were operated under anaesthesia. The main procedures were hernioplasty/herniorrhaphy, resection and anastomosis, adhesiolysis and colostomy/ileostomy with or without resection of pathological bowel. The operative findings, surgical procedure done and operative diagnosis were noted. The pathological diagnosis, in cases where specimen/tissue sample sent for histopathological analysis, were recorded. The postoperative period was monitored carefully. The patients were followed up till discharge or death and any morbidity or mortality factors were also recorded.

The data obtained are compiled, tabulated, analysed using appropriate statistical methods according to age, sex, symptoms, signs, X-ray abdomen (Erect) findings, operative procedure done, aetiological diagnosis, post-operative complications and mortality, along with its causes.

\section{RESULTS}

The age range in the present study was from 14 years to 82 years. (Table 1) The maximum incidence was seen in 41-50 years $(25.24 \%)$, followed by $50-60$ years $(20 \%)$. The mean age in this study was 46.7 years. Male: Female ratio was 2.68:1.

\begin{tabular}{|c|c|c|c|c|}
\hline $\begin{array}{c}\text { Age Group } \\
\text { (In Years) }\end{array}$ & Male & Female & Total & \% \\
\hline$\leq 20$ & 10 & 5 & 15 & $7.14 \%$ \\
\hline $21-30$ & 14 & 5 & 19 & $9.05 \%$ \\
\hline $31-40$ & 25 & 10 & 35 & $16.67 \%$ \\
\hline $41-50$ & 39 & 14 & 53 & $25.24 \%$ \\
\hline $51-60$ & 31 & 11 & 42 & $20.00 \%$ \\
\hline $61-70$ & 22 & 10 & 32 & $15.24 \%$ \\
\hline $71-80$ & 11 & 1 & 12 & $5.71 \%$ \\
\hline$>80$ & 1 & 1 & 2 & $0.95 \%$ \\
\hline Total & $\mathbf{1 5 3}$ & $\mathbf{5 7}$ & $\mathbf{2 1 0}$ & $\mathbf{1 0 0}$ \\
\hline \multicolumn{6}{|c}{ Table 1. Age \& Sex Distribution } \\
\hline
\end{tabular}

Pain abdomen (89.05\%), constipation (84.76\%), abdominal distension (78.57\%) and vomiting (76.19\%) were the predominant symptoms. X-ray abdomen (Figure 1) erect was helpful in majority of cases of obstruction.

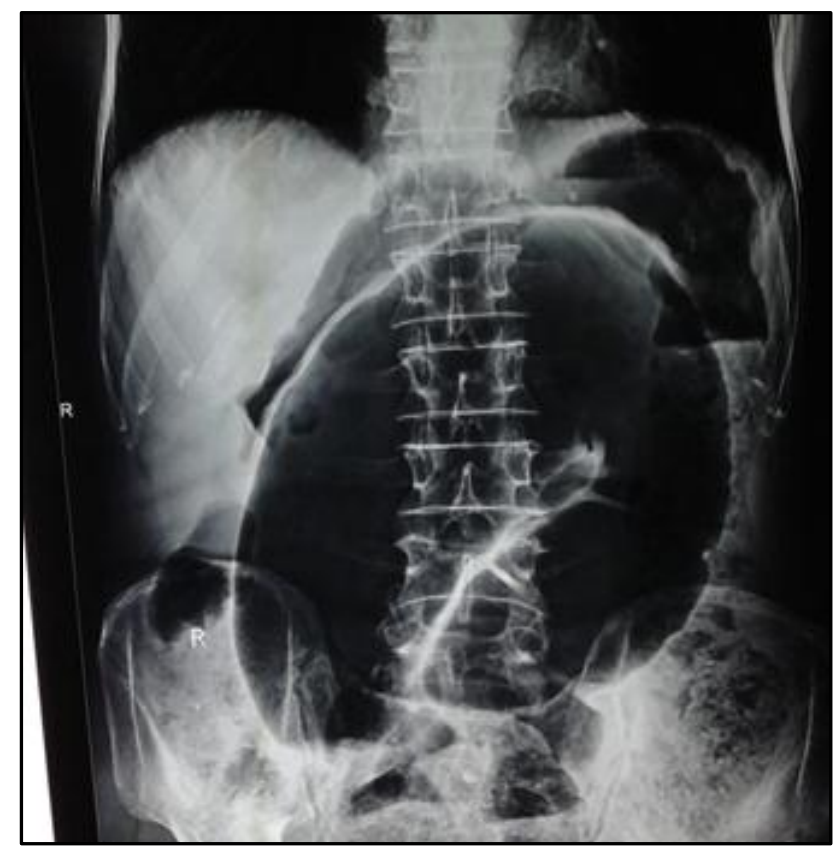

Figure 1. X-ray showing Sigmoid Volvulus

The most common cause of intestinal obstruction in this setup was external hernias (31.43\%), followed by adhesions $(25.24 \%)$ and volvulus (20\%). Carcinoma of colon was seen 
in $12.85 \%$. The most common aetiology in males was obstructed abdominal wall hernias (34.9\%) followed by adhesions. In females, both adhesions and carcinoma colon constitute $24.6 \%$ each of the causes of obstruction, hence were the major causes of obstruction. (Figure $2 \& 3$ ).

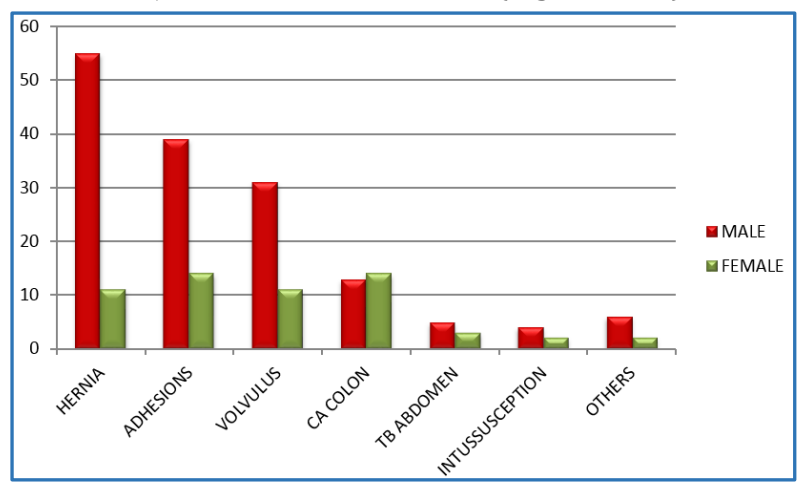

Figure 2. Aetiological Distribution

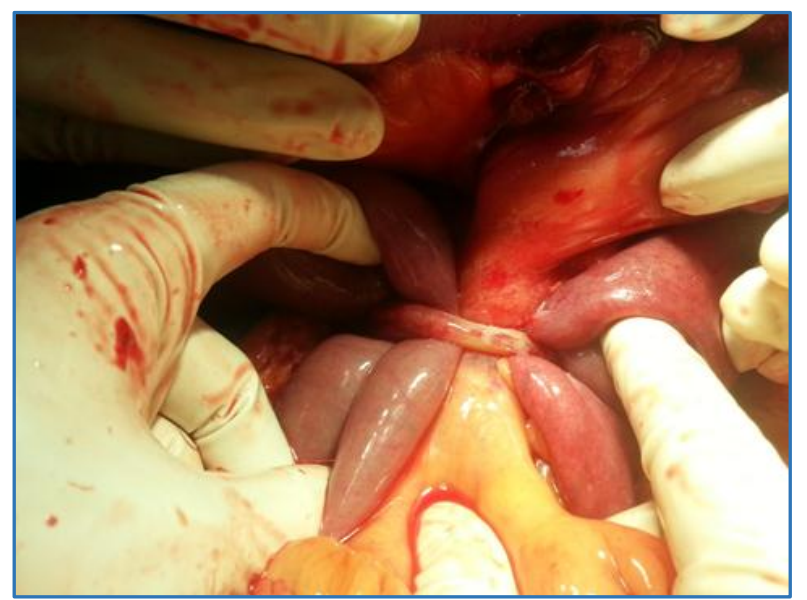

Figure 3. Intraoperative View of Band causing Intestinal Obstruction

Bands and adhesions (33.3\%) and carcinoma colon $(33.3 \%)$ were the most common causes for acute intestinal obstruction below 20 years of age. The main cause in 21-40 age group was adhesions (29.6\%) followed by volvulus (24.1\%). The most common aetiology in older age groups i.e. 41-60 (37.9\%) \& 61-80 (47.7\%) were obstructed external hernias followed by bands and adhesions (26.3\%) and volvulus (29.5\%), respectively.

In majority of the cases, small bowel was involved (64.76\%), whereas large bowel was involved in 33.81\%.

Resection and anastomosis was the most common surgical procedure done (43.81\%), followed by adhesiolysis (22.86\%) and hernioplasty (21.43\%). (Figure 4).

\section{SURGICAL PROCEDURE PERFORMED}

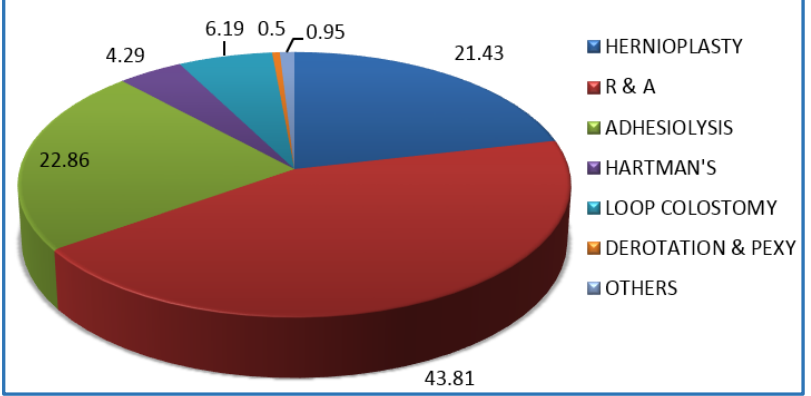

Figure 4. Surgical Procedure Performed
In the postoperative period, 43 cases $(20.47 \%)$ developed complications. Wound infection $(10.47 \%)$ was the most common followed by respiratory infection (7.14\%), enterocutaneous fistula (2.86\%) and burst abdomen (4.29\%). Majority of the cases who developed wound infection (86.4\%), respiratory infection $(100 \%)$, burst abdomen $(100 \%)$ and enterocutaneous fistula (83.3\%) were above 40 years of age. Males were more commonly having wound infection $(77.3 \%)$, burst abdomen $(60 \%)$ and respiratory infections (93.3\%) when compared to females. Enterocutaneous fistula was seen in males and females in equal proportion. Resection was done in majority of cases which developed postoperative wound infection (81.8\%) and burst abdomen (80\%). Resection was not done in $50 \%$ of cases who developed fistula in the postoperative period. The main aetiologies causing morbidity in postoperative period includes adhesions (39.47\%), obstructed hernias (36.84\%) and sigmoid volvulus (23.68\%).

The mortality rate in the current study was $8.09 \%$. The main causes of mortality were carcinoma colon (23.5\%), acute mesenteric ischaemia (17.6\%), sigmoid volvulus $(17.6 \%)$ and compound volvulus (17.6\%). The causes having high mortality rates were acute mesenteric ischaemia and ileosigmoid knotting (75\% in each pathology). The main cause of mortality in majority of cases (52.3\%) was septicaemia which leads on to multiorgan failure. Most of the deaths $(88.2 \%)$ occurred in first postoperative week.

\section{DISCUSSION}

Intestinal obstruction occurs in all age groups. In the present study, $45.24 \%$ belong to $41-60$ years of age group, followed by $21-40$ years age group (25.72\%) and $61-80$ years age group $(20.95 \%)$. This was comparable to studies done by Souvik A et al and Viji DGK.1,5 Male to female ratio was 2.68:1, which is similar to pattern seen in studies by Souvik et al, Viji DGK, Sinha $S$ et al and Ramrao BS et al.1,5,6,7 The gender discrepancy in the patients with males outnumbering females by a huge margin can be possibly accounted for, as a large number of the patients had obstructed inguinal hernia and sigmoid volvulus, and in our country, mostly males suffer from these conditions.

The cardinal features in intestinal obstruction, i.e. pain abdomen (89.05\%), constipation (84.76\%), abdominal distension (78.57\%) and vomiting (76.19\%) were seen in majority of cases in this study. It was comparable to various studies done by Khan JS et al, Madziga AG et al, Thampi D et al and Malik AM et al.8,9,10,11 The minor differences noted may be due to the difference in time of presentation to hospital in various setups.

The causes of acute intestinal obstruction vary with geographical locations. Obstructed external hernia (31.43\%) was the most common aetiology in this study followed by adhesions (25.24\%). This is because patients are reluctant for elective surgery of hernias due to poverty, lack of education and general fear of surgery. Significant regional variation in the aetiology of intestinal obstruction has been noted amongst various Indian studies. This pattern of aetiology of obstruction was seen in most of the developing countries. It was seen in various Indian studies by Souvik A et al, Viji DGK and Khan JS et al.1,5,8 Study done by Madziga AG et al in Nigeria and Ntakiyiruta $G$ et al in Rwanda were showing similar results. ${ }^{9,12}$ Sigmoid volvulus $(20 \%)$ was the third 
common cause of obstruction which produces ischaemia and necrosis of the colon very early due to twisting of mesentery. The incidence of sigmoid volvulus was more in the present study than the other studies. This is because majority of current study population are from villages and they used to take high fibre diet, predisposing to sigmoid volvulus. Small intestine was the site of obstruction in $64.76 \%$ and large intestine in 33.81\%. In studies by Ramrao BS et al, Malik AM et al, Mohamed AY et al and Markogiannakis $\mathrm{H}$ et al, more of small intestinal obstructions were seen.7,11,13,14 This difference is mostly because volvulus involving the sigmoid colon was seen in a major group (20\%) in the present study. (Table 2).

\begin{tabular}{|c|c|c|c|c|c|c|c|}
\hline Aetiology & $\begin{array}{c}\text { Present } \\
\text { Study }\end{array}$ & $\begin{array}{c}\text { Viji } \\
\mathbf{D G K}^{\mathbf{5}}\end{array}$ & $\begin{array}{c}\text { Thampi D } \\
\text { et al }{ }^{\mathbf{1 0}}\end{array}$ & $\begin{array}{c}\text { Souvik } \\
\text { et al }\end{array}$ & $\begin{array}{c}\text { Ntakiyiruta G } \\
\text { et al } \mathbf{1}^{\mathbf{2}}\end{array}$ & $\begin{array}{c}\text { Madziga AG } \\
\text { et al }^{\mathbf{9}}\end{array}$ & $\begin{array}{c}\text { Khan JS } \\
\text { et al }^{\mathbf{8}}\end{array}$ \\
\hline Hernia & $31.43 \%$ & $37 \%$ & $30 \%$ & $35.96 \%$ & $39 \%$ & $35.0 \%$ & $34 \%$ \\
\hline Adhesions & $25.24 \%$ & $22 \%$ & $40 \%$ & $15.53 \%$ & $17.1 \%$ & $26.61 \%$ & $25 \%$ \\
\hline Volvulus & $20 \%$ & $12 \%$ & $4 \%$ & $6.26 \%$ & $10.5 \%$ & $2.95 \%$ & $6 \%$ \\
\hline Carcinoma Colon & $12.85 \%$ & $14 \%$ & $14 \%$ & $16.621 \%$ & - & $9.14 \%$ & $3 \%$ \\
\hline TB Abdomen & $3.8 \%$ & $3 \%$ & $4 \%$ & $14.17 \%$ & - & - & - \\
\hline \multicolumn{8}{|c|}{ Table 2. Comparison of Aetiologies in Various Studies } \\
\hline
\end{tabular}

Wound infection $(10.47 \%)$ was the most common postoperative complication in the present study followed by respiratory tract infection (7.14\%). This is in accordance with various studies by Souvik et al, Ramrao BS et al and Madziga AG et al, which shows that wound infection is the most common cause of morbidity in postoperative cases of intestinal obstruction.1,7,9 The other major complications were respiratory tract infection, burst abdomen and enterocutaneous fistula. Wound infection was more commonly seen in cases where adhesiolysis was done, than in cases where resection was done for adhesions. This might be due to the prolonged surgical procedure taking more than 3 hours in cases of adhesiolysis and the antibiotics given was probably not adequate for that. Enterocutaneous fistula in cases of postoperative adhesions was more commonly seen in cases where adhesiolysis was done than resection. This might be because, at the points of adhesiolysis, damage to seromuscular coat would have resulted in fistula formation. If there is any suspicion that seromuscular coat is devitalised, it needs to be inverted with seromuscular stitches or resected and re-anastomosed.

The mortality rate in the present study was $8.09 \%$. It is comparable with mortality rates in various studies by Souvik et al (7.35\%), Viji DGK (9\%), Ramrao BS et al (6.2\%) and Madziga AG et al (9.14\%).1,5,7,9 Poor outcomes have been noted in patients with early development of bowel gangrene like compound volvulus (sigmoid volvulus with ileosigmoid knotting) and acute mesenteric ischaemia and also in cases of malignancies. The rate of bowel gangrene has been reported to be $93-100 \%$ in ileosigmoid knotting. The mortality rate in cases of acute mesenteric ischaemia was very high where 3 out of 4 cases died in this study. This is similar to the pattern seen in study by Nonthasoot B et al. ${ }^{15}$

\section{CONCLUSION}

Acute intestinal obstruction still remains a major cause of morbidity and mortality. The pattern of obstruction is changing with increasing adhesions and decreasing hernias. Both the sexes were affected with a male preponderance. The maximum incidence occurred in 41-60 years of age group. Pain abdomen, vomiting, abdominal distension and constipation were seen in majority of cases. Intravenous fluids, nasogastric aspiration, broad spectrum antibiotics and correction of electrolyte imbalances before surgery were as important as the surgery itself and hence remain the mainstay of initial treatment. Plain x-ray abdomen (Erect posture) was the main diagnostic investigation in cases of acute intestinal obstruction. It was having better accuracy in cases of distal obstructions. The most common cause of obstruction was obstructed external hernias (31.43\%), followed by adhesions (25.24\%) and sigmoid volvulus (20\%). Adhesions were the most common aetiology below 40 years and above that age it was obstructed hernias. Sigmoid volvulus also forms a major share of cases causing acute intestinal obstruction in the present study. The most common site of obstruction was the small intestine (64.76\%). Small intestinal obstruction was mostly caused by obstructed hernias and adhesions, whereas large intestinal obstruction was by sigmoid volvulus and colonic malignancies. Resection and anastomosis was the most common surgery performed in the present study, followed by adhesiolysis and hernioplasty. Intra-operative findings varied from simple bands to malignancy. The most common postoperative complications were wound infection, respiratory tract infection, enterocutaneous fistula and burst abdomen. Resection was done in majority of cases which developed wound infection and burst abdomen. The main aetiologies causing morbidity were adhesions, obstructed hernias and sigmoid volvulus. Morbidity was seen more commonly in elderly patients. Mortality was seen in $8.09 \%$. The causes having high mortality rates were acute mesenteric ischaemia and ileosigmoid knotting.

Ileosigmoid knotting and acute mesenteric ischaemia have to be taken care of as these causes are difficult to diagnose early and are having high mortality rates. The diagnosis of strangulation is still a challenge. Prompt intervention in cases of strangulation is the key for a better outcome. Even though there are great advances in technology at the investigation level, clinical judgement based on history, physical examination and radiography of the abdomen forms the main part in diagnosing a case of acute intestinal obstruction. For affected patients, high quality surgical expertise coupled with sound clinical judgement and early surgery when needed will greatly improve the survival. Sustained efforts at elective hernia repair and research aimed at reducing adhesions are likely to reduce the high morbidity and mortality from acute intestinal obstruction. Furthermore, a general improvement in health infrastructure especially in rural areas could further reduce mortality as patients may present early. 


\section{REFERENCES}

[1] Souvik A, Hossein MZ, Amitabha D, et al. Etiology and outcome of acute intestinal obstruction: a review of 367 patients in Eastern India. Saudi J Gastroenterol 2010;16(4):285-7.

[2] Sclabas GM, Sarosi GA, Khan S, et al. Small bowel obstruction. In: Zinner MJ, Ashley SW, (eds). Maingot's abdominal operations. $12^{\text {th }}$ edn. New York: McGraw-Hill Company ltd 2013:585-610.

[3] Osuigwe AN, Anyanwu SNC. Acute intestinal obstruction in Nnewi, Nigeria: a five-year review. Nigerian Journal of Surgical Research 2002;4(3):10711.

[4] Tavakkoli A, Ashley SW, Zinner MJ. Small intestine. In: Brunicardi FC, Anderson DK, Billiar TR, (eds). Schwartz's principles of surgery. 9th edn. New York: McGraw-Hill education 2015:1137-74.

[5] Viji GK. A prospective clinical study of dynamic intestinal obstruction in a tertiary care centre. Indian Journal of Applied Research 2016;6(5):111-3.

[6] Sinha S, Kaushik R, Yadav TD, et al. Mechanical bowel obstruction: the Chandigarh experience. Trop Gastroenterol 2002;23(1):13-5.

[7] Ramrao BS, Ekanath JS, Marutirao NA. A prospective study of intestinal obstruction in a rural hospital in India. Indian Journal of Applied Research 2012;1(12):166-8.
[8] Khan JS, Alam J, Hassan H, et al. Pattern of intestinal obstruction: a hospital based study. Pak Armed Forces Med J 2007;57(4):295-9.

[9] Madziga AG, Nuhu AI. Causes and treatment outcome of mechanical bowel obstruction in north eastern Nigeria. West Afr J Med 2008;27(2):101-5.

[10] Thampi D, Tukka VN, Bhalki N, et al. A clinical study of surgical management of acute intestinal obstruction. Int J Res Health Sci 2014;2(1):299-308.

[11] Malik AM, Shah M, Pathan R, et al. Pattern of acute intestinal obstruction: is there a change in the underlying etiology? Saudi J Gastroenterol 2010; 16(4):272-4.

[12] Ntakiyiruta G, Mukarugwiro B. The pattern of intestinal obstruction at Kibogora hospital, a rural hospital in Rwanda. East \& Central African Journal of surgery 2009;14(2):103-8.

[13] Mohamed AY, al-Ghaithi A, Langevin JM, et al. Causes and management of intestinal obstruction in a Saudi Arabian hospital. J R Coll Surg Edinb 1997;42(1):21-3.

[14] Markogiannakis H, Messaris E, Dardamanis D, et al. Acute mechanical bowel obstruction: clinical presentation, etiology, management and outcome. World J Gastroenterol 2007;13(3):432-7.

[15] Nonthasoot B, Tullavardhana T, Sirichindakul B, et al. Acute mesenteric ischemia: still high mortality rate in the era of 24-hour availability of angiography. J Med Assoc Thai 2005;88( Suppl 4):S46-50. 